\title{
Assessing out-of-band flare effects at the wafer level for EUV lithography
}

\author{
Simi A. George, Patrick P. Naulleau, Charles D. Kemp, Paul E. Denham, and Senajith Rekawa \\ Center for X-Ray Optics, Lawrence Berkeley National Laboratory, Berkeley, CA 94720 \\ Corresponding author, Email: sageorge@lbl.gov
}

\begin{abstract}
To accurately estimate the flare contribution from the out-of-band (OOB), the integration of a DUV source into the SEMATECH Berkeley 0.3-NA Micro-field Exposure tool is proposed, enabling precisely controlled exposures along with the EUV patterning of resists in vacuum. First measurements evaluating the impact of bandwidth selected exposures with a table-top set-up and subsequent EUV patterning show significant impact on line-edge roughness and process performance. We outline a simulation-based method for computing the effective flare from resist sensitive wavelengths as a function of mask pattern types and sizes. This simulation method is benchmarked against measured OOB flare measurements and the results obtained are in agreement.
\end{abstract}

Keywords: EUVL, out-of-band, resists, flare, blur on patterns, aerial image modeling

\section{INTRODUCTION}

Pulsed plasma based EUV sources generated from high-Z metallic elements radiate into a broad range of wavelengths; well beyond the $13.5 \mathrm{~nm}$ wavelength at the $2 \%$ bandwidth required for EUV lithography (EUVL). ${ }^{1-2}$ Calculations and measurements have shown that the Mo-Si multilayer based reflective optics necessary for band selection at EUV are also equally or more efficient reflectors for a range of wavelengths longer than $150 \mathrm{~nm}$ (figure 1, left). In addition to this, the EUV resist development methods utilize existing molecular platforms already developed for ultraviolet (UV) and deep ultraviolet (DUV) wavelength lithography, leading to the sensitivity of these resists to a group of wavelengths between $150 \mathrm{~nm}$ and $300 \mathrm{~nm}$ (figure 1, right). The combined effect of all of these factors is the unwanted background exposure of the resist, resulting in reduced imaging fidelity. This effective flare contribution from OOB, in addition to the flare resulting from the low spatial frequency roughness scatter of the optics, is expected to be significant.
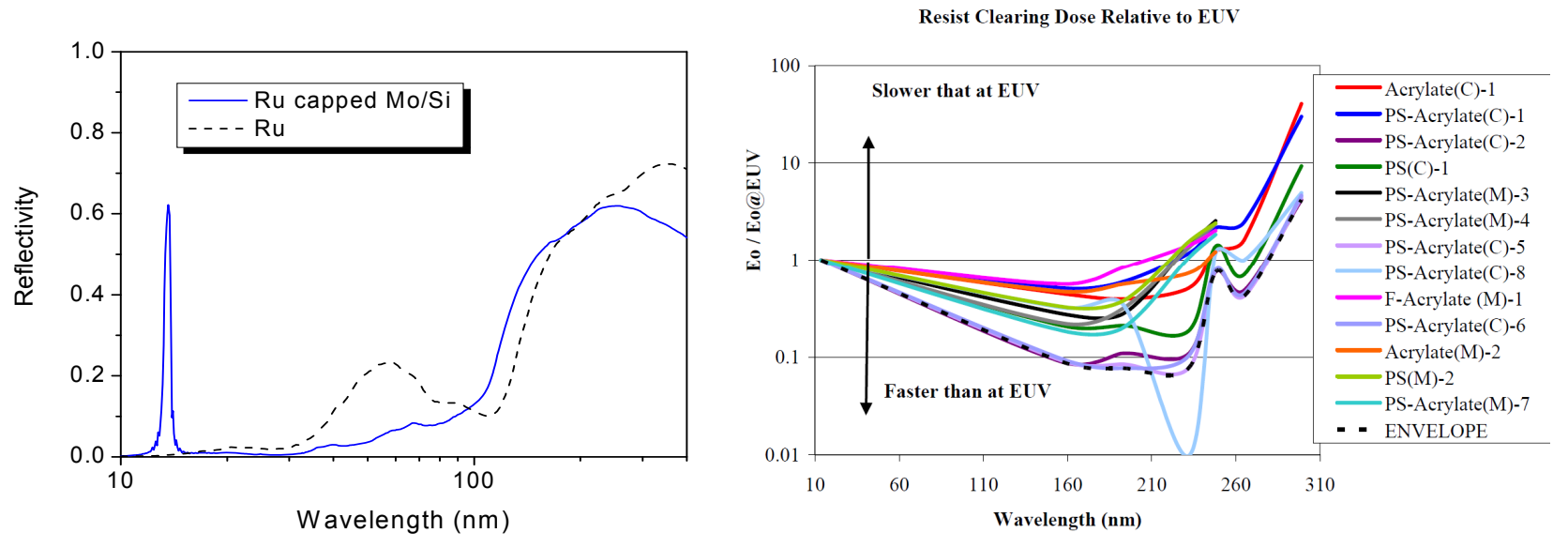

Figure 1. (Left) figure shows the near normal incidence ( 5 degrees) for Ru and for a standard Ru capped Mo/Si multilayer. The reflectivity was calculated using optical constants from the Handbook of Optical Constants of Solids ${ }^{3-4}$ (Data courtesy of Dr. Eric Gullikson). (Right) Chemically amplified resists ${ }^{5}$ sensitivity measured for the UV/DUV wavelengths. Almost all platforms tested are shown to be more sensitive to DUV/UV wavelengths than at EUV. ${ }^{6}$ 
In this paper, a basic method for the first order estimation of effective flare from the resist sensitive out of band (OOB) wavelengths is outlined. This method is benchmarked against previously published measurements results from a fullfield exposure tool. Results from the first set of controlled OOB experiments are also presented.

\section{OOB RELATED FLARE ESTIMATIONS AT THE IMAGE PLANE}

A simple method for estimating DUV/UV contribution from sources in addition to the EUV dose at the image plane was outlined previously. ${ }^{7}$ Resist dependant flare was calculated with supplier provided OOB wavelength specific resist sensitivity values as compared to the published absolute EUV sensitivity ${ }^{8}$ for a resist. This particular resist was more sensitive to $157 \mathrm{~nm}$ radiation than for other wavelengths. The effective fare was calculated by dividing the $100-400 \mathrm{~nm}$ region into four bands. A summary of the complete data set is provided in table 1 . The starting point for all calculations is the pseudo focal point of the lithography exposure optical system called the intermediate focus. ${ }^{9}$ Depending on the design the number of mirrors utilized for projection and imaging may vary and estimates for a 10 and 11 mirror system are given in table 1. Depending on the resist platforms utilized, the absorption of OOB wavelengths will vary ${ }^{6}$ and this may lead to considerable reduction in patterning fidelity.

Table 1: Effective flare calculated from OOB wavelength specific resist sensitivity as related to the EUV sensitivity

\begin{tabular}{|c|c|c|c|c|c|c|}
\hline $\begin{array}{l}\text { Wavelength } \\
\text { (nm) }\end{array}$ & $\begin{array}{l}\mathrm{E}_{0} \text { Rel. Sens. } \\
\left(\mathrm{mJ} / \mathrm{cm}^{2}\right)\end{array}$ & $\begin{array}{l}\text { Sensitivity } \\
\text { Factor }\end{array}$ & $\begin{array}{l}11 \text { Mirrors, } \\
10 \% \text { OOB, } \\
\text { throughput } \\
\text { from IF }(\%)\end{array}$ & $\begin{array}{l}11 \text { Mirrors, } \\
10 \% \text { OOB } \\
\text { at IF, resist } \\
\text { dependent } \\
\text { flare }(\%)\end{array}$ & $\begin{array}{l}10 \text { Mirrors, } \\
10 \% \text { OOB, } \\
\text { throughput } \\
\text { from IF }(\%)\end{array}$ & $\begin{array}{l}10 \text { Mirrors, } \\
10 \% \text { at IF, } \\
\text { resist } \\
\text { dependent } \\
\text { flare }(\%)\end{array}$ \\
\hline 13.5 & 1.9 & $1 \mathrm{x}$ & 100 & 100 & 100 & 100 \\
\hline 157 & 0.5 & $4 \mathrm{x}$ & 0.79 & 3.15 & 0.93 & 3.74 \\
\hline 193 & 7.9 & $0.24 \mathrm{x}$ & 1.46 & 0.35 & 1.74 & 0.42 \\
\hline 248 & 2.2 & $1 \mathrm{x}$ & 0.83 & 0.83 & 1.01 & 1.01 \\
\hline 365 & $\gg 13.5 \mathrm{~nm}$ & Negligible & 0.29 & Negligible & 0.37 & Negligible \\
\hline \multicolumn{4}{|c|}{ Total Effective OOB flare on wafer (Ru capped MLM) } & 4.34 & & 5.17 \\
\hline
\end{tabular}

Traditionally, flare from OOB is treated as a uniform background (DC) problem. This may not be completely accurate, since the shorter wavelengths in the DUV can image the large features on the mask. Furthermore, it can be expected that the effective flare and the resulting contrast loss for patterning may vary with feature types, sizes, density, and with the tone of the mask. In order to evaluate such effects, a simple in-house developed, two dimensional point spread function (PSF) model is utilized.

\subsection{ADT flare test features modeled as a benchmark}

Recently measurements were completed at the ASML alpha demo tool (ADT) in order to observe and estimate the flare contribution from OOB at the wafer level. ${ }^{10}$ Varying number of reflections from reticle masking blades for OOB exposures were used while imaging a circular absorber post of $200 \mathrm{~nm}$ diameter surrounded by a bright area on the mask. The diameter of the bright area was varied, while the absorber diameter was kept constant. It was found that the contributions from flare increased for larger bright area diameters. The measurements led to the estimate that there is approximately $4 \%$ OOB radiation at the image plane.

In order to benchmark the modeling methodology, these measurements are simulated. The multilayer and absorber reflectivities for the $13.5 \mathrm{~nm}$ central wavelength and the three key wavelengths $(157 \mathrm{~nm}, 193 \mathrm{~nm}, 248 \mathrm{~nm})$ were computed using the optical constants listed in table 2 and using the Stack program available from Panoramic technology. ${ }^{11} \mathrm{~A}$ multilayer reflectivity of $64 \%$ and an absorber reflectance of $2.0 \%$ was calculated and these values were used for the PSF mask modeling. ADT numerical aperture of 0.25 and circular illumination with a pupil factor of $0.5(\sigma)$ were used for the aerial image calculations. For the OOB aerial image calculations, an average multilayer reflectivity of $74 \%$, and an absorber reflectivity of $16 \%$ were used. Images were calculated assuming incoherent illumination. Examples of the masks generated for use with the PSF model are shown in figure 2. Bright area diameters from $250 \mathrm{~nm}$ to $5 \mu \mathrm{m}$ are used around a $200 \mathrm{~nm}$ circular absorber post. Figure 3 shows the calculated images for different wavelengths of interest, starting with the $13.5 \mathrm{~nm}$ illumination. A drop in image intensity and image contrast is observed as the illumination wavelength is increased. 
Table 2. Optical constants used for determining the multilayer and absorber reflectivities

\begin{tabular}{|c|c|c|c|c|c|}
\hline & $\mathbf{1 3 . 5} \mathbf{~ n m}$ & $\begin{array}{c}\mathbf{1 5 7} \mathbf{~ n m} \\
\mathbf{( 7 . 8 9 7} \mathbf{e V})\end{array}$ & $\begin{array}{c}\mathbf{1 9 3} \mathbf{~ n m} \\
\mathbf{( 6 . 4 2 4} \mathbf{e V})\end{array}$ & $\begin{array}{c}\mathbf{2 4 8} \mathbf{~ n m} \\
\mathbf{( 5 . 0 0 0} \mathbf{~ e V})\end{array}$ & $\begin{array}{c}\mathbf{3 6 5} \mathbf{~ n m} \\
\mathbf{( 3 . 3 9 7} \mathbf{e V})\end{array}$ \\
\hline Materials & $\mathrm{n}, \mathrm{k}$ & $\mathrm{n}, \mathrm{k}$ & $\mathrm{n}, \mathrm{k}$ & $\mathrm{n}, \mathrm{k}$ & $\mathrm{n}, \mathrm{k}$ \\
\hline *TaN & $\mathrm{N} / \mathrm{A}$ & $1.53,0.97$ & $1.89,1.29$ & $2.49,1.59$ & $3.22,1.28$ \\
\hline *TaBN(Absorber) & $0.950,0.0289$ & $\mathrm{~N} / \mathrm{A}$ & $2.80,1.76$ & $2.60,1.81$ & $\mathrm{~N} / \mathrm{A}$ \\
\hline$* *$ TaON (ARC) & $0.954,0.0260$ & $\mathrm{~N} / \mathrm{A}$ & $1.93,0.88$ & $2.60,0.40$ & $\mathrm{~N} / \mathrm{A}$ \\
\hline$* * *$ Amorph.-Si & $0.999,0.0018$ & $0.66,1.60$ & $0.96,2.07$ & $1.69,2.76$ & $3.90,2.66$ \\
\hline$* * * \mathrm{Mo}$ & $0.924,0.0064$ & $0.70,1.77$ & $0.80,2.36$ & $1.46,3.62$ & $3.06,3.19$ \\
\hline$* * * \mathrm{Ru}$ & $0.887,0.0168$ & $0.66,1.50$ & $0.81,2.04$ & $1.03,2.70$ & $1.84,4.30$ \\
\hline
\end{tabular}

*Approximated for TaON, TaBN absorber $\operatorname{stack}^{12},{ }^{* *}$ Measured data ${ }^{13},{ }^{* * *}$ From IMD optical constants database by David Windt ${ }^{14}$

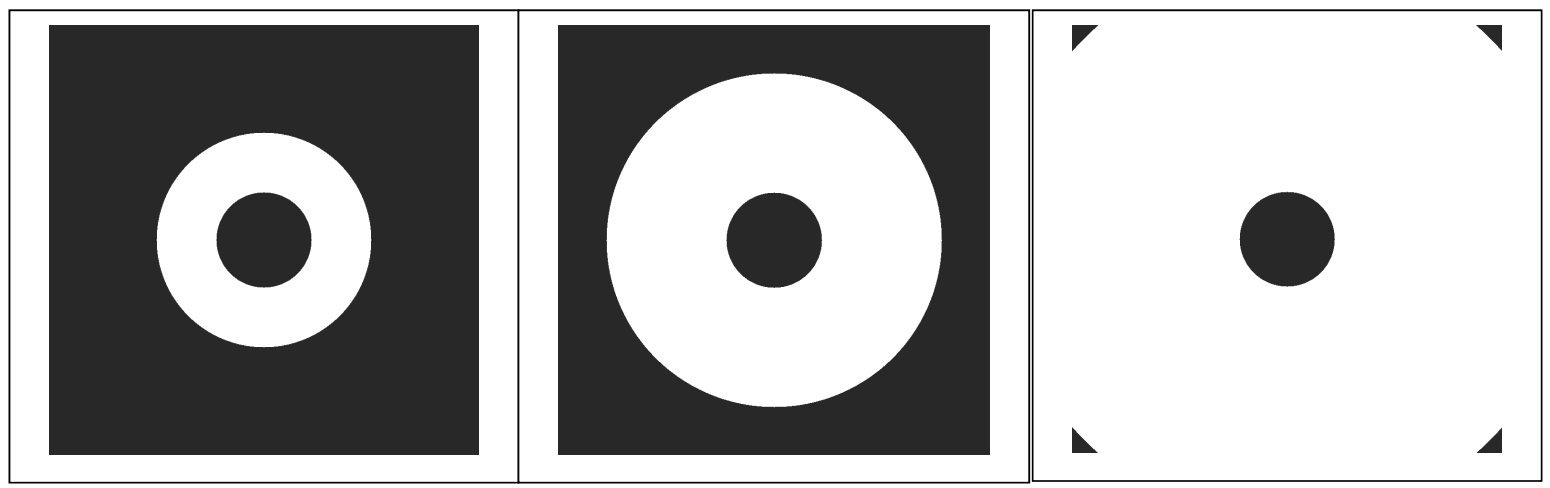

Figure 2. Flare test features generated with varying bright area diameters $(250 \mathrm{~nm}, 500 \mathrm{~nm}, 1000 \mathrm{~nm})$ to use with 2D PSF aerial image model (image space). The circular absorber post in the middle has a diameter of $200 \mathrm{~nm}$ and kept constant for all mask patterns.
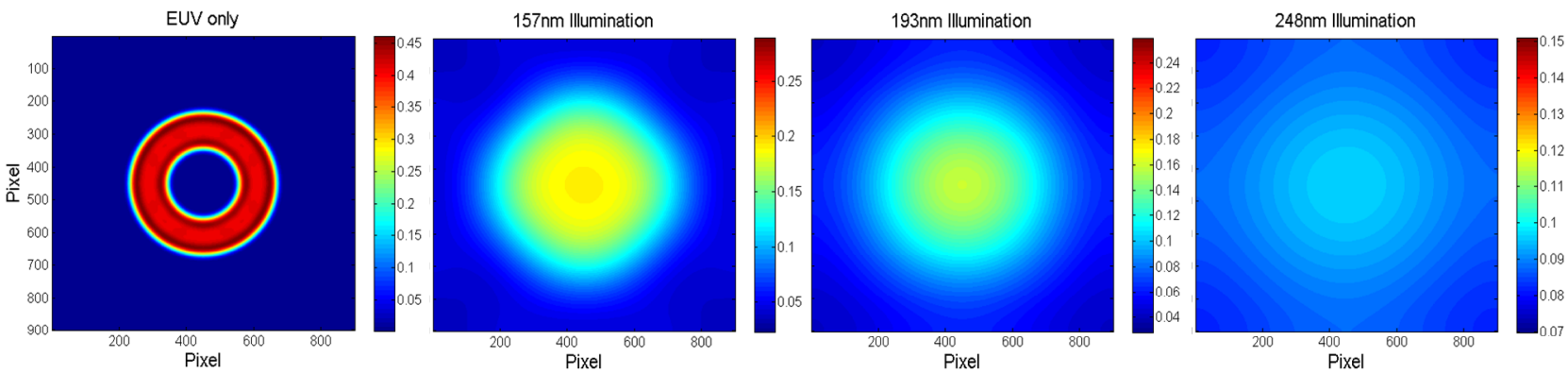

Figure 3. 2D PSF model calculated aerial images for a flare test feature with $250 \mathrm{~nm}$ bright area surrounding a $200 \mathrm{~nm}$ absorber post with the different illumination wavelengths.

In order to produce the OOB imaging effect over the EUV image, the fraction of the image intensities is obtained in accordance with the resist dependent percent contributions given in table 1 and added to the EUV aerial image. The resulting image for the 11 mirror system assumption is shown in figure 4 (left), with a light halo produced from the OOB contribution over the EUV image. Figure 4 (right) shows the cross section of the OOB included images for the 10- and 11- mirror systems as compared to the pure EUV aerial image. Effective flare contribution is calculated from these intensity profiles using the simple formulation based on the Kirk flare: ${ }^{15}$

$$
\text { Effective flare }=\left(\frac{E U V_{\min }}{E U V_{\max }}\right)-\left(\frac{U V_{\min }}{U V_{\max }}\right)
$$

The effective flare values calculated in this way for the different bright area flare features are tabulated in table 3 .

Lowest flare is found for the smallest bright area diameter, supporting the ADT measurements. A plot of these tabulated values is shown in figure 5. A saturation effect is found as the bright areas become much larger than the $200 \mathrm{~nm}$ post feature. 

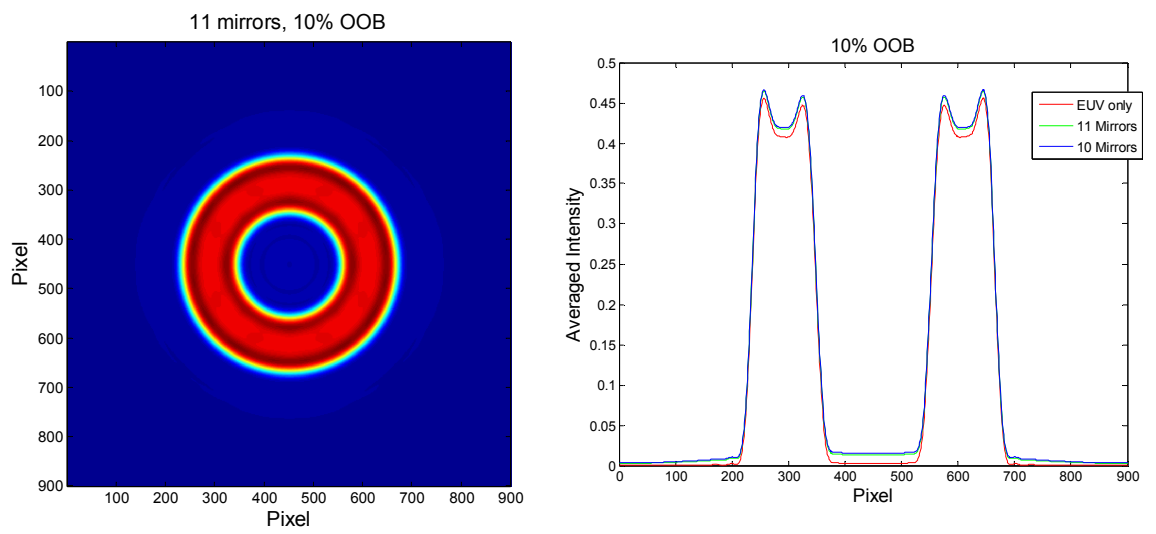

Figure 4. OOB effects over image, produced by adding the resist dependent image intensity fractions of the long wavelength aerial images to the EUV image (left). Image intensity profiles for the pure EUV image along with image profiles of OOB added images for the 10 and 11 mirror systems (right).

Table 3. Tabulated flare contribution from OOB, for features with varying bright area rings around a 200nm absorber post

\begin{tabular}{|c|c|c|c|c|}
\hline & \multicolumn{2}{|c|}{$10 \%$ OOB } & \multicolumn{2}{c|}{$20 \%$ OOB } \\
\hline \multirow{3}{*}{ Bright Area Diameter (nm) } & \multicolumn{3}{|c|}{ Flare (\%) on the 200nm circular post } \\
\cline { 2 - 5 } & $\begin{array}{c}11 \\
\text { mirrors }\end{array}$ & $\begin{array}{c}10 \\
\text { mirrors }\end{array}$ & $\begin{array}{c}11 \\
\text { mirrors }\end{array}$ & $\begin{array}{c}10 \\
\text { mirrors }\end{array}$ \\
\hline 5000 & 3.91 & 4.63 & 7.42 & 8.71 \\
\hline 1000 & 3.53 & 4.19 & 6.68 & 7.85 \\
\hline 500 & 3.12 & 3.73 & 6.00 & 7.07 \\
\hline 250 & 2.68 & 3.18 & 5.24 & 6.19 \\
\hline
\end{tabular}

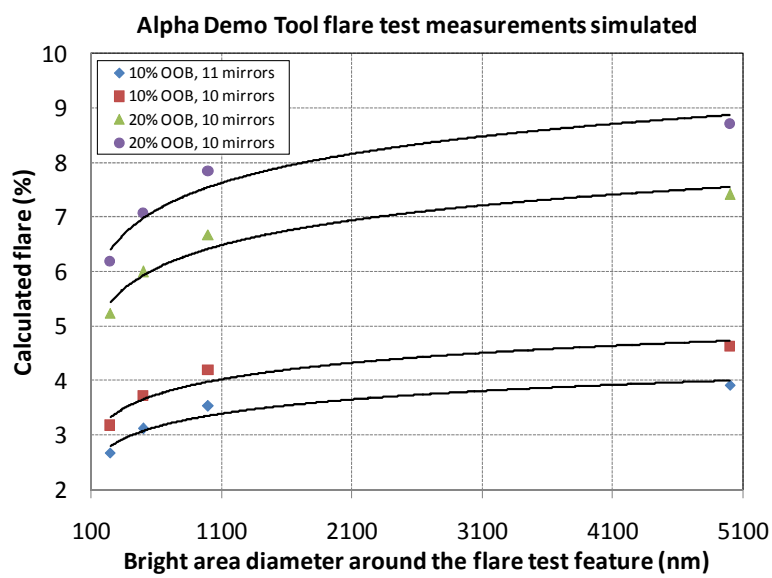

Figure 5. Plot of the tabulated OOB flare (table 3). Saturation in the flare may be expected as the bright areas become infinitely large in comparison to the $200 \mathrm{~nm}$ absorber post. A very large bright area was not calculated for computational reasons.

The model outlined here is believed to give a first order approximation of the effective flare on feature resulting from the longer, resist sensitive wavelengths reaching the wafer plane. It is worth noting that these values are estimated based on one set of resist parameters and neglecting any other effects such as mask scattering, changes in absorber reflectivity, diffraction effects, pitch dependencies, etc. Since all of the aerial image simulations are completed in image space and since these features are much larger in comparison to the critical dimensions required, it may be that we are under estimating the flare effects. The next section outlines results for several mask features under varying conditions. 


\subsection{Feature specific flare estimates with the 2D model}

Making use of the same methods described in the previous section, feature specific evaluations are completed for flare effects at the $22 \mathrm{~nm}$ half pitch. Absorber effects are examined with the bright and dark clear mask areas on isolated and dense, line and contact features. Typical production type system parameters with $0.32 \mathrm{NA}, 0.5 \sigma$ and conventional illumination are used. Figure 5 gives examples of the mask types for the $22 \mathrm{~nm}$ line/space CD. Significant differences in contrast are observed between the bright area mask and the dark area mask. Values computed for the different features are tabulated in table 4. It is shown that flare on features does not directly translate to smaller features. Dense features show modulation effects and drastic variations in contrast depending on the feature types. Some mitigation of flare from absorber masks is seen. Although, we assume that diffraction effects and 3D effects contribute at this level, calculations that account for these are not completed at this time.
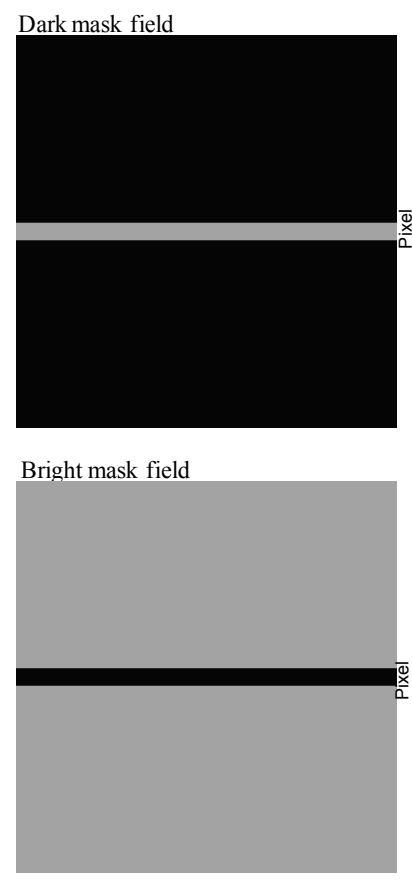

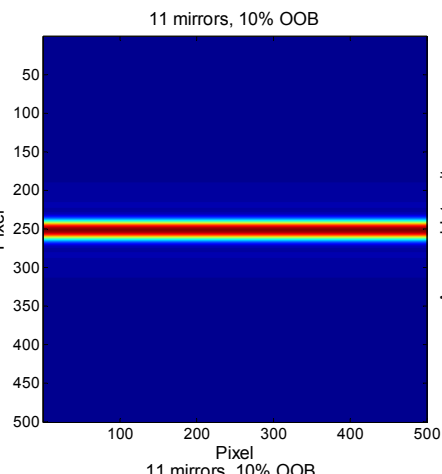

11 mirrors, $10 \% \mathrm{OOB}$

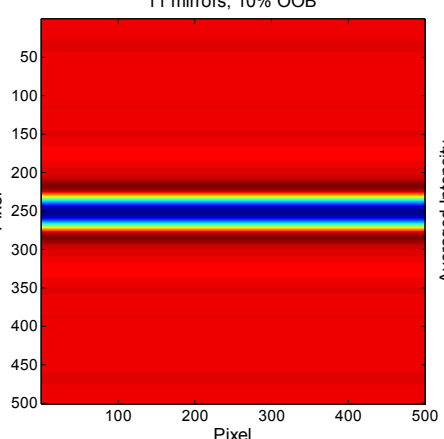

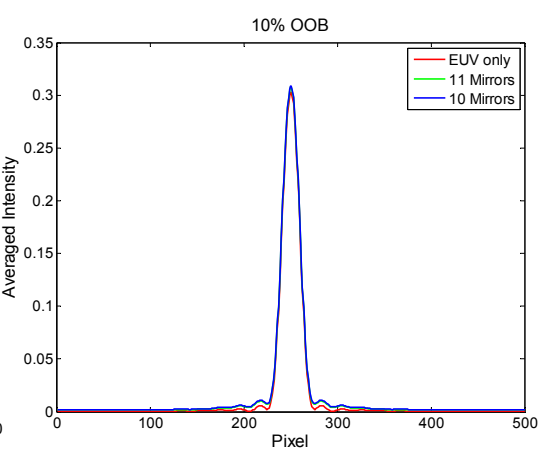

$10 \% \mathrm{OOB}$

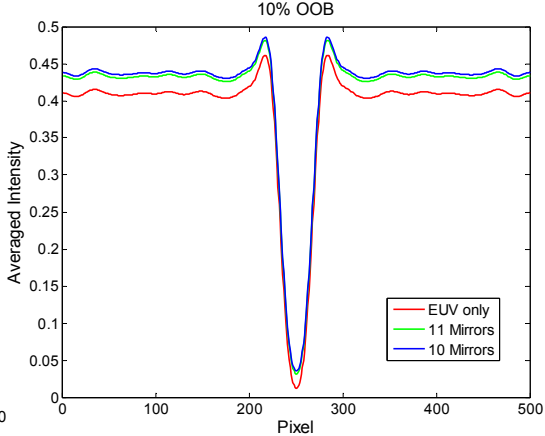

Figure 5. OOB effects over EUV image for a $22 \mathrm{~nm}$, isolated bright and dark line. Intensity profiles significant differences in the two cases as compared with the flare on the dark line is more double that of the dark mask/bright line case.

Table 4. Flare contributions from OOB wavelengths for different features with $22 \mathrm{~nm}$ critical dimension

\begin{tabular}{|c|c|c|}
\hline & \multicolumn{2}{|c|}{$\mathbf{1 0} \%$ OOB } \\
\hline & \multicolumn{2}{|c|}{ Effective flare on features (\%) } \\
\hline Optics Train & $\mathbf{1 1}$ mirrors & $\mathbf{1 0}$ mirrors \\
\hline Dense Patterns & 7.23 & 8.62 \\
\hline Bright contacts & 5.04 & 6.01 \\
\hline Dark lines & 3.68 & 4.40 \\
\hline Bright lines & 2.22 & 2.65 \\
\hline Elbows & 1.72 & 2.05 \\
\hline Isolated Patterns & 5.42 & 6.48 \\
\hline Bright Line & 2.19 & 2.61 \\
\hline Dark Line & 5.68 & 7.23 \\
\hline Bright Contact &
\end{tabular}




\section{OOB EXPOSURES AT THE MICRO-EXPOSURE TOOL}

The SEMATECH Berkeley 0.3 NA, EUV micro-field exposure tool (MET) installed at the Advanced Light Source synchrotron facility at Lawrence Berkeley National Laboratory currently uses a spectrally pure, debris-free, undulator radiation as the source of EUV radiation. The MET uses programmable coherence illumination ${ }^{16}$ and provides imaging capabilities down to $12 \mathrm{~nm}$, enabling advanced resist, mask, process, and metrology methods development. Further details on the MET can be found in the literature ${ }^{17}$. The proposed experimental plan for enabling precisely controlled OOB imaging studies with MET involves incorporating a DUV/UV source of sufficient brightness and uniformity at a point in the MET optical train.

Figure 6 (left) shows the mechanical design for incorporating the LDLS source into MET. Optical design was completed using ZEMAX and toroidal mirrors are utilized for light collection and beam shaping. The mirrors are diamond turned (for low rms surface roughness), Nickel plated blanks from Welch Mechanical Designs coated with Aluminum in-house. Aluminum was found to have the best performance in terms of the calculated reflectivity with good linearity for the wavelengths of interest. The source is to be inserted above the turning mirror for the synchrotron illumination, bypassing the fly's eye mirrors of the MET and passing through the scanning illuminator. The $1 \mathrm{~mm}$ aperture at the source provides a source etendue small enough to support the MET NA requirements. This beam line integration of DUV source is in the process of being completed.

\subsection{Out-of-Band Exposure Methods}

Current OOB exposure set-up does not directly incorporate the MET. The DUV light source is set-up on an optical bench and radiation is focused onto resist coated 4-inch wafers. The calibrated, spectral distribution from this source for available wavelengths of interest to this study is shown in figure 6 (right) for a $1 \mathrm{~mm}$ aperture at source output. The measured power output at the two key resist sensitive wavelengths of $193 \mathrm{~nm}$ and $248 \mathrm{~nm}$ are $0.04 \mathrm{~mW} / \mathrm{nm}$ and 0.09 $\mathrm{mW} / \mathrm{nm}$, respectively. Wavelength selection for the exposures is implemented with filters obtained from Acton Optics \& Coatings. ${ }^{18}$ These filters have approximately $24 \mathrm{~nm}$ of FWHM bandpass. Filters are aligned into the optical path and interchanged using a fly wheel. This way controlled resist exposures can be completed at the three illumination configurations; the $193 \mathrm{~nm}$ band, the $248 \mathrm{~nm}$ band, and in the broadband.
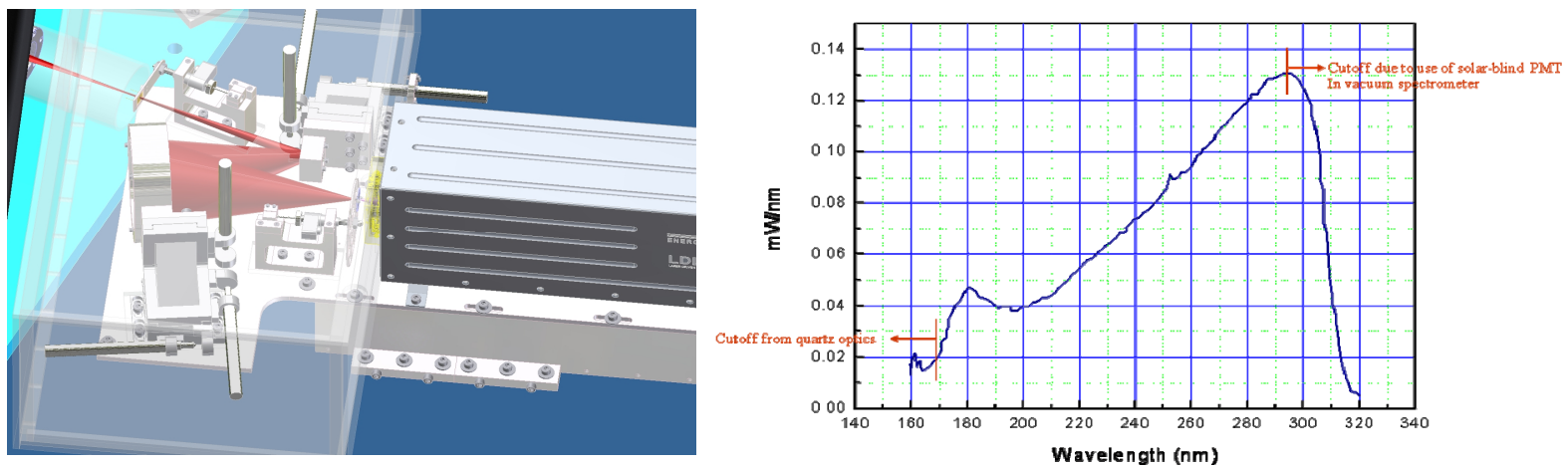

Figure 6. (Left) Current table top set-up for the pre-exposure of resists with bandwidth selected OOB wavelengths. (Right) Source spectral output provided by the source supplied. Wavelengths from 170-290nm are available.

For exposures, the DUV beam is collected, focused, and apertured to a uniform $8 \mathrm{~mm}$ X 10mm area. An SXUV IRD photodiode ${ }^{18}$ with an active area of $10 \mathrm{~mm} \times 10 \mathrm{~mm}$ is used for dose measurements. Nearly linear responsivity of this photodiode to the DUV wavelengths makes it ideal for these measurements. Dose measurements are taken prior to each exposure to monitor any possible changes. The resist coated wafer is aligned to the DUV beam inside a high purity Nitrogen purged chamber and exposed. The exposed wafer is removed and placed into a desiccator and vacuum sealed for transport to MET. Care is taken to minimize any contact of the samples to amine or oxygen rich environment after DUV exposures. EUV patterning is completed over the DUV exposed area on the wafer with keeping all process conditions as similar as possible.

For these initial set of experiments, a baseline, chemically amplified EUV resist was evaluated. Film thickness used for the resist coating on Hexamethyldisilazane primed Silicon wafer is $50 \mathrm{~nm}$. Measurements comparing $193 \mathrm{~nm}$ band 
illumination and the broadband illumination related performance effects on this resist are completed. The exposure dose for both illuminations is kept as close as possible to $1 \mathrm{~mJ} / \mathrm{cm}^{2}$. Exact uncertainty in the exposure dose is not available at this time.

\subsection{Results: $193 \mathrm{~nm}$ bandwidth selected exposures}

The exposed and developed resist patterns are imaged using the Hitachi ultra-high resolution field emission scanning electron microscope model S- $4800 .{ }^{19} \mathrm{SEM}$ images of $40 \mathrm{~nm}$ half-pitch vertical lines data was collected for a number of exposure steps through focus. Figure 7 shows images collected for the resist patterns with and without $193 \mathrm{~nm}$ illumination. The top three images are obtained for dose and focus optimized 40nm half-pitch vertical lines for the EUV only reference imaging condition. An average $3 \sigma$ LER of $4.8 \mathrm{~nm}$ was found for these lines. Bottom three images are the best images obtained for EUV patterning over the $193 \mathrm{~nm}$ exposed area on resist. It should be noted that these images are obtained from two different wafers, where one will not have seen any OOB at all. By visual inspection of the images it is immediately obvious that LER is increased. The second set of images produced an average $3 \sigma$ LER of $7.2 \mathrm{~nm}$ after analysis.
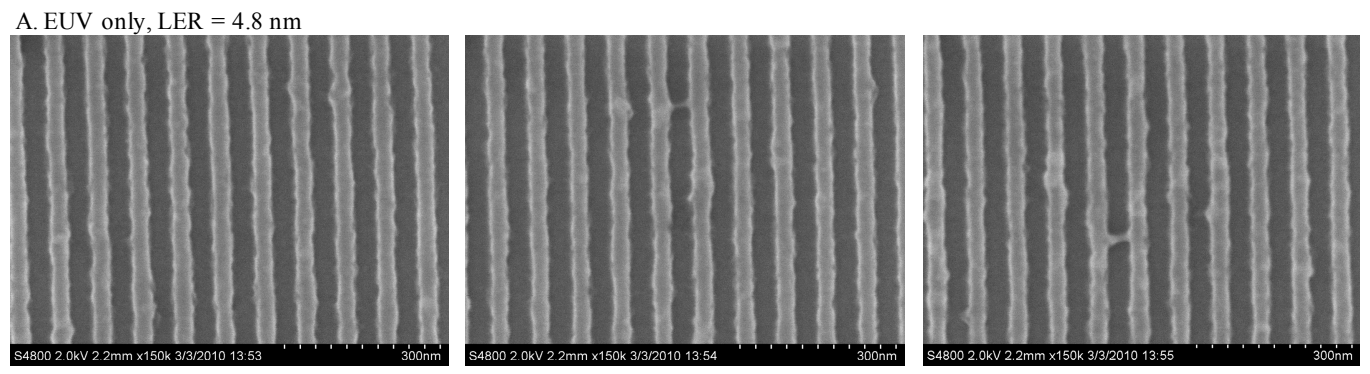

B. EUV imaging over $193 \mathrm{~nm}$ Illumination
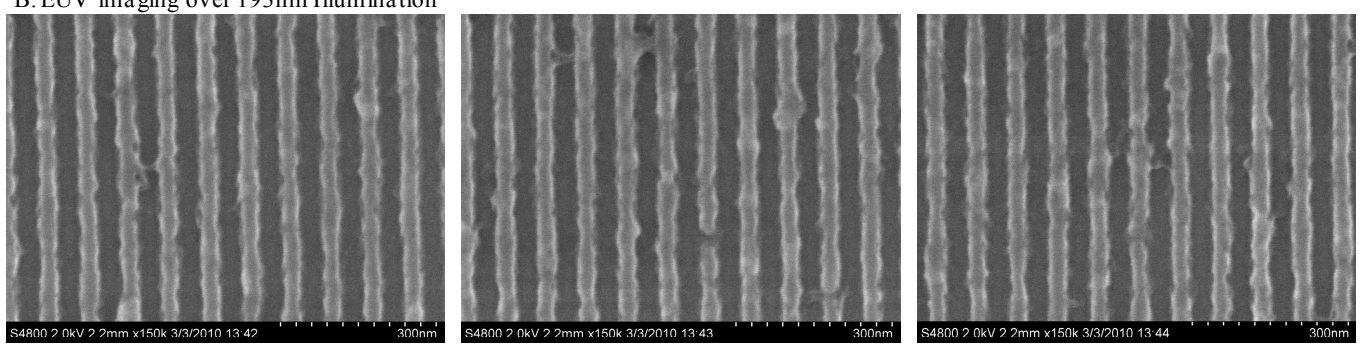

Figure 7. A) Images collected at the best dose and focus for the 1:1 40nm half pitch vertical lines for EUV only patterning. Average LER of $4.8 \mathrm{~nm}$ was found for these lines. B) Best images obtained for EUV patterning over the 193nm exposed area on resist, with an average $3 \sigma$ LER of $7.2 \mathrm{~nm}$.
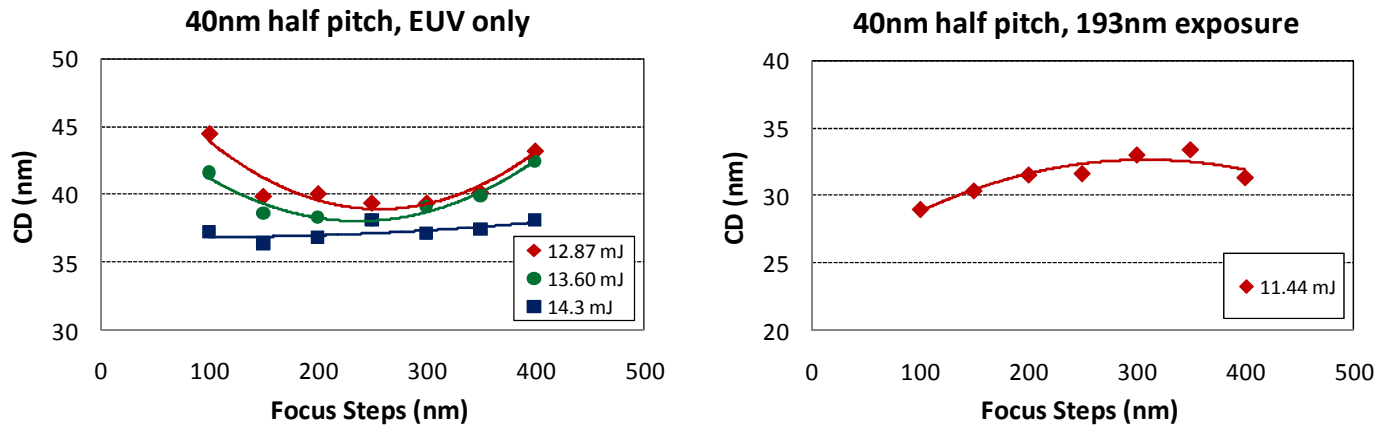

Figure 8. (Left) Through focus and dose LER distribution from EUV only patterning for the 248nm optimized polymer platform for $40 \mathrm{~nm}$ vertical 1:1 patterns. (Right) Best data collected for $193 \mathrm{~nm}$ illuminated patterning.

Detailed image analysis of process performance and line edge roughness (LER) measurements are completed with the commercially available software, SuMMit. ${ }^{20}$ Through focus and dose LER distribution from EUV only patterning for the $248 \mathrm{~nm}$ optimized polymer platform is shown in figure 8 (left). Best 40nm vertical 1:1 patterns are found for a EUV 
dose of $12.87 \mathrm{~mJ}$. In comparison, the best 40nm lines were obtained for the first EUV exposure step over the 193nm illumination. The collected data appear to be overdosed and behaves more like the $15.0 \mathrm{~mJ}$ pure EUV exposure. We expect that the absorbance of this resist for $193 \mathrm{~nm}$ radiation is very high and enough to shift the EUV dose size significantly. The LER corresponding to the collected lines and spaces data is shown in figure 9, for both conditions. LER is significantly increased for $193 \mathrm{~nm}$ band illuminated patterning as was shown previously.
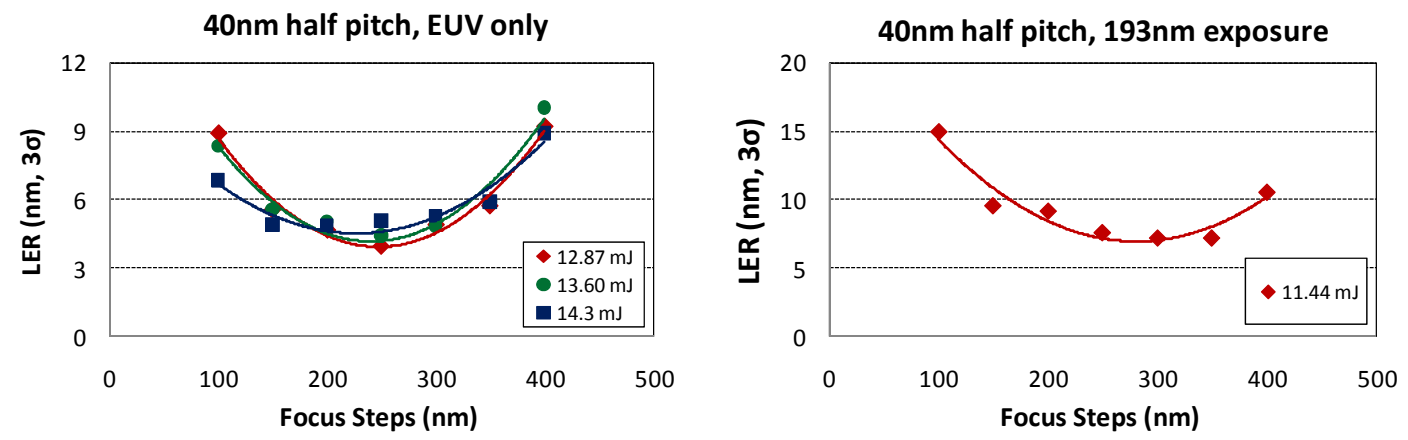

Figure 9. 3б LER measurements corresponding to the process data shown in figure 8 (Left) EUV only imaging, (Right) EUV patterning over $193 \mathrm{~nm}$ illumination dose of $1 \mathrm{~mJ} / \mathrm{cm}^{2}$

\subsection{Unfiltered, broad-band exposures}

Previous measurements were repeated for the same resist under the same conditions but with $1 \mathrm{~mJ} / \mathrm{cm}^{2}$ broadband illumination. 40nm half-pitch lines and spaces 1:1 pattern process is compared to EUV only exposures of the same. Figure 10 shows process data obtained for both. Interestingly, the broad-band illuminated data (10, right) show a clear isofocal region at $40 \mathrm{~nm}$ with extended depth of focus and a slightly decreased EUV exposure dose. Process performance seemed to have improved with broadband illumination.
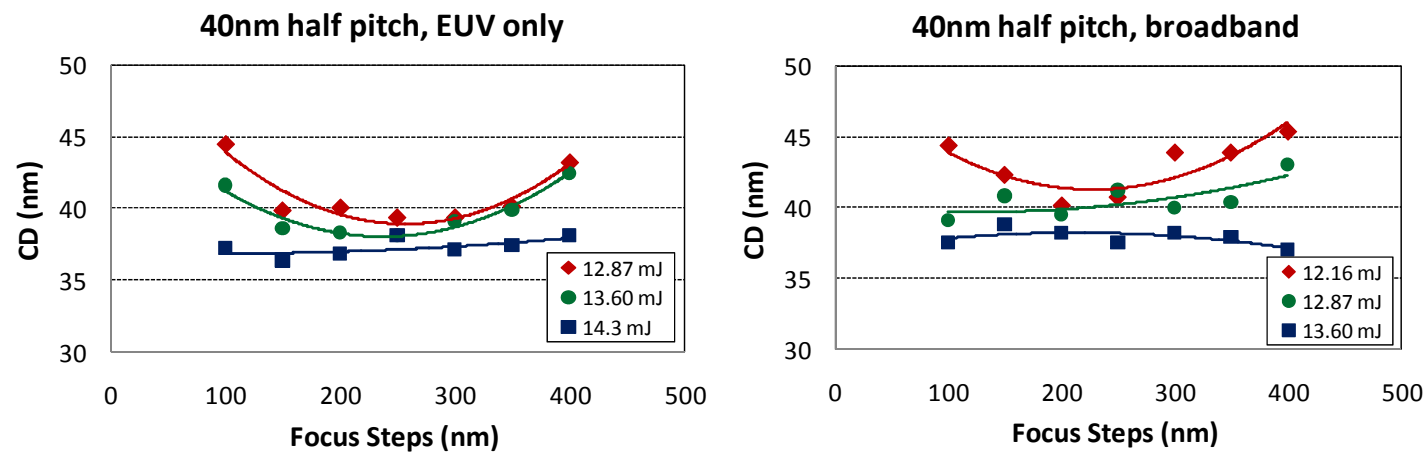

Figure 10. (Left) Through focus and dose LER distribution from EUV only patterning for the 248nm optimized polymer platform for $40 \mathrm{~nm}$ vertical 1:1 patterns. (Right) EUV patterning over a broadband illumination of $1 \mathrm{~mJ} / \mathrm{cm}^{2}$.

In looking at the corresponding LER data, the LER at the defocused regions of the process window show significant increase for the broadband illuminated patterns (figure 11, right). An average LER of $5.2 \mathrm{~nm}$ was found for the best conditions, while the EUV only patterning gave an average LER of $4.8 \mathrm{~nm}$. The reason behind the improved process is understood clearly at this time. Further examination of the resolution performance is needed as well. Rigorous examination of the induced chemical changes in the film as a result of UV exposure needs to be better understood. 

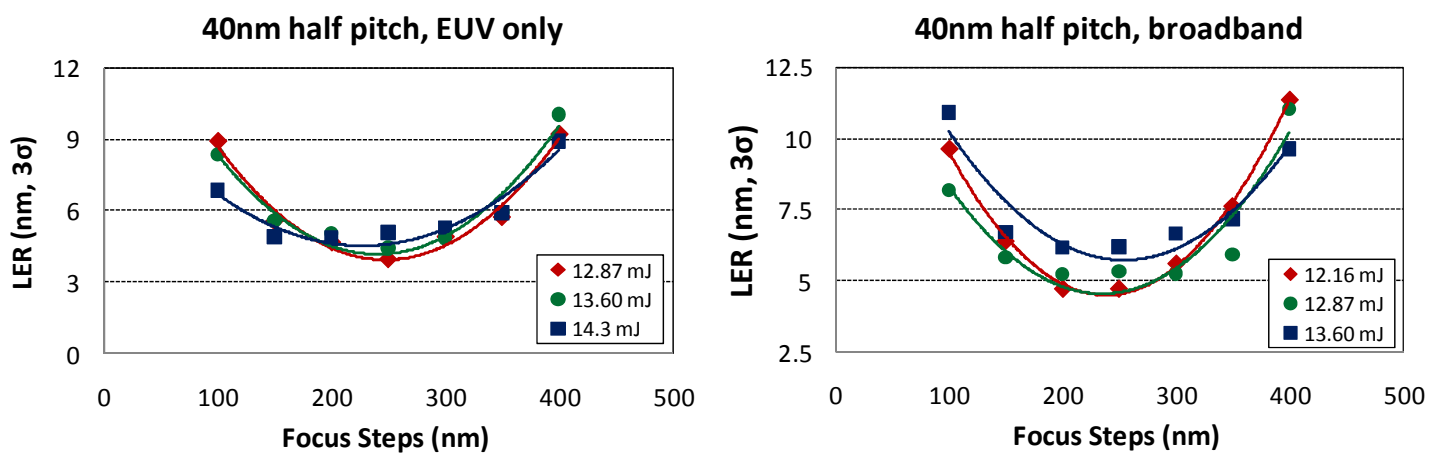

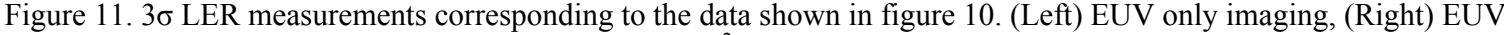
patterning over a broadband illumination dose of $1 \mathrm{~mJ} / \mathrm{cm}^{2}$, average LER of $5.2 \mathrm{~nm}$ was found for the best conditions.

\section{SUMMARY AND DISCUSSION}

In this paper, a basic method for the first order estimation of effective flare from the resist sensitive out of band (OOB) wavelengths is outlined. This method is benchmarked against previously published measurements results from the fullfield ASML ADT lithography exposure tool. The simulation results are in agreement with the measurements.

Results from the first set of controlled OOB experiments are also presented. The results show an impact on LER and on process performance. Possible chemical changes induced by VUV light may involve the removal of lactone and ester groups in resist and changes in the glass transition temperature induced by VUV heating. Efforts are underway for a more rigorous and complete experimental evaluation of multiple resists.

\section{ACKNOWLEDGMENTS}

The authors would like to acknowledge the support and dedication of the CXRO engineering team and staff. Special thanks to the MET team members Brian Hoef, and Gideon Jones. Mirror reflectance and optical constants were provided by Dr. Eric Gullikson. We would like to acknowledge the Energetiq team, especially Debbie Gustafson and Robert Angeli, for providing support and source spectral characterizations for this work. This work was supported by the Director, Office of Science, Office of Basic Energy Sciences, of the U.S. Department of Energy under contract number DE-AC02-05CH11231.

\section{REFERENCES}

[1] Bakshi, V., ed., "EUV Sources for Lithography," SPIE Press, Washington (2005)

[2] Stulen, R. and Sweeney, D., "Extreme ultraviolet lithography," Quantum Electronics, IEEE Journal of 35, 694-6999 (1999).

[3] Gullikson, E., "Multilayer reflectivity," (2007).

[4] Palik, E. D. and Ghosh, G., Handbook of Optical Constants of Solids, Academic Press, San Diego, CA (1997). http://henke.lbl.gov/optical constants.

[5] Thackeray, J. W., Nassar, R. A., Brainard, R., Goldfarb, D., Wallow, T., Wei, Y., Mackey, J., Naulleau, P., Pierson, B., and Solak, H. H., Chemically amplified resists resolving $25 \mathrm{~nm}$ 1:1 line: space features with euv lithography," Emerging Lithographic Technologies XI 6517(1), 651719, SPIE (2007).

[6] Roberts, J. M. Bristol, R. L., Younkin, T. R., Fedynyshyn, T. H., Astolfi, D. K., and Cabral, A., "Sensitivity of EUV resists to out-of-band radiation,” Proc. SPIE 7273, 72731W (2009). 
[7] George, S. A., Naulleau, P. P., Rekawa, S., Gullikson, E., and Kemp, C. D., "Estimating the out-of-band radiation flare levels for extreme ultraviolet lithography," J. Micro/Nanolith. MEMS MOEMS 8, 041502 (2009)

[8] Naulleau, P. P., Gullikson, E. M., Aquila, A., George, S., and Niakoula, D., Absolute sensitivity calibration of extreme ultraviolet photoresists," Opt. Express 16(15), 11519-11524 (2008)

[9] Miyake, A., Kanazawa, H., Banine, V., and Suzuki, K., Joint requirements," Presentation at EUV Work-shop, Proceedings available at www.sematech.org (October 19. 2006).

[10] Mizuno, H., McIntyre, G., Koay, C-S., Burkhardt, M., LaFontaine, B., and Wood, O., "Flare evaluation of ASML alpha demo tool," Proc. SPIE 7271, 72710U (2009)

[11]Panoramic Technology, "EM-Suite/Hyperlith," http://www.panoramictech.com (2009).

[12] Optical Properties of Thin Films for DUV and VUV Microlithography, Nanolithography research labs, http://www.rit.edu/kgcoe/microsystems/lithography/thinfilms/thinfilms/thinfilms.html

[13] Email communication, PN Naulleau and Seh-Jin park, September 24, 2008.

[14] IMD - Software for modeling the optical properties of multilayer films', D. L. Windt, Computers in Physics, 12, 360 (1998)

[15] Murakami, K., Oshino, T., Kondo, H., Chiba, H., Nomura, K., Kawai, H., Kohama, Y., Morita, K., Hada, K., Ohkubo, Y., and Miura, T., "Development of EUV lithography tools at Nikon," Proc. SPIE 7140, 71401C (2008)

[16] Naulleau, P. P., Anderson, C. N., Chiu, J., Dean, K., Dehnam, P. E., George, S. A., Goldberg, K. A., Hoef, B. H., Jones, G., Koh, C., La Fontaine, B., Ma, A., Montgomery, W., Niakoula, D., Park, J.-O., Wallow, T. I., Wurm, S. "Latest results from the SEMATECH Berkeley extreme ultraviolet mircofield exposure tool," JVST B, 27, p. 66, (2009)

[17] Acton Optics \& Coatings, “Optical filters," (2009). http://www.princetoninstruments. com/products/optics/filters/default.aspx\#1.

[18] SXUV Series Photodiodes, http://www.ird-inc.com/sxuv.html

[19] Hitachi High-Technologies, http://www.hht-eu.com/cms/3171.html

[20] EUV Technology SuMMIT Software Division, http://www.lithometrix.com 